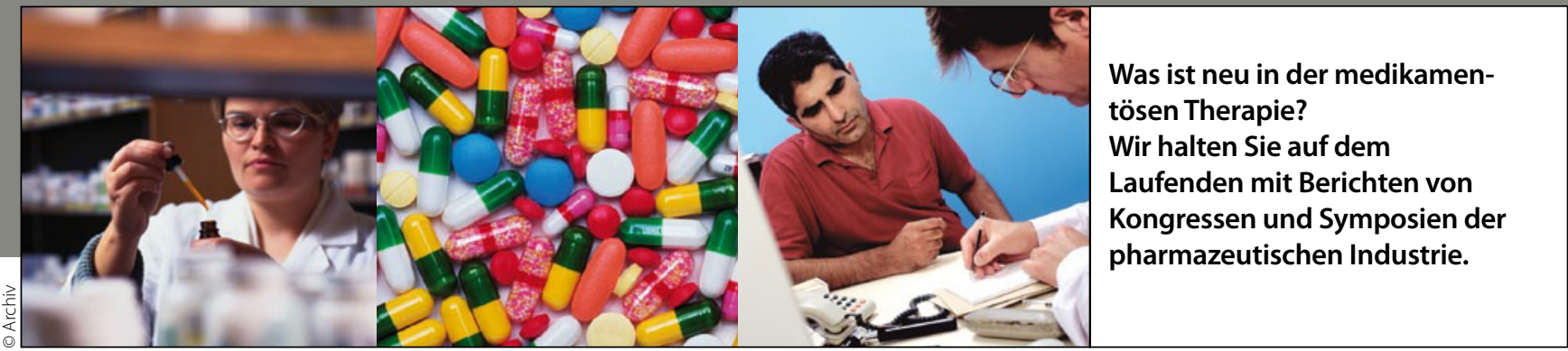

Renin-Inhibitor plus Kalziumantagonist

\title{
Neue Fixkombination verspricht besonderen Organschutz
}

- Neu auf dem Markt ist die Fixkombination Aliskiren/Amlodipin gegen Bluthochdruck. Ideale Patienten dafür sind solche mit Diabetes, Übergewicht und/oder einer Nierenschädigung, erklärte der Hypertonieexperte Dr. Ralf Dechend, Berlin Buch.

Zwei Drittel aller Hochdruckpatienten benötigen Kombinationstherapien. Die europäischen Hypertonie-Leitlinien empfehlen zur Steigerung der Compliance „wenn immer möglich“ den Einsatz von Fixkombinationen, deren Fundament ein Blocker des Renin-Angiotensin-Systems sein sollte. Dies gilt insbesondere für Diabetiker.

Gerade bei hypertensiven Diabetikern mit Übergewicht ist die Kombination eines RAAS-Blockers mit einem Kalziumantagonisten, idealerweise mit Amlodipin, sinnvoll. Dies hat die ACCOMPLISH-Studie gezeigt. Im Vergleich zur Kombi ACEHemmer/HCT reduzierte die Kombination ACE-Hemmer/Amlodipin das Risiko für kardiovaskuläre Komplikationen um 20\%.

\section{Renaler Blutfluss gesteigert}

Der direkte Renininhibitor Aliskiren hat gegenüber anderen RAAS-Blockern den Vorteil, dass er die Plasma-Renin-Aktivität (PRA) nicht erhöht, sondern senkt. Die PRA gilt als ein unabhängiger Risikofaktor für die kardiovaskuläre und renale Morbidität. Auch lokale RAAS-Systeme werden durch Aliskiren effektiv gesenkt. Dies könnte zu einem gegenüber $\mathrm{ACE}-\mathrm{Hemmern}_{\mathrm{oder}} \mathrm{AT}_{1}{ }^{-}$ Blockern überlegenen Endorganschutz führen und prognostische Vorteile mit sich bringen, so Dechend. Nach den Daten der AVOID-Studie wirkt Aliskiren renoprotektiv.
Bei Diabetes sind die RAAS-Systeme besonders überaktiv. Bekannt ist, dass lokale RAAS-Systeme in der Niere die diabetische Nephropathie fördern. Aliskiren wirkt hier stark inhibitorisch und steigert den renalen Blutfluss deutlich mehr als ACE-Hemmer oder AT $_{1}$-Blocker, berichtete Dechend.

\section{Starke Blutdrucksenkung}

Darüber hinaus wirkt der direkte Renininhibitor stark blutdrucksenkend und ist dabei sehr gut verträglich. Die Fixkombination Aliskiren/Amlodipin senkte in einer achtwöchigen Doppelblindstudie den Blutdruck signifikant stärker als Amlodipin allein $(-38 / 16 \mathrm{mmHg}$ vs. $-31 / 12 \mathrm{mmHg})$. Bei schwerer Hypertonie ist der additive Effekt auf den systolischen Blutdruck noch ausgeprägter (- $47 \mathrm{mmHg}$ vs. $-37 \mathrm{mmHg}$ ). Im Vergleich zu einer Kombinationsbehandlung Olmesartan/Amlodipin 40/10 $\mathrm{mg} / \mathrm{d}$ zeigte sich Aliskiren/Amlodipin 300/10 mg/d überlegen wirksam (systolische RR-Senkung - 26 mmHg vs. - 21 $\mathrm{mmHg}$ ).

Sowohl Aliskiren (40 Std.) als auch Amlodipin (50 Std.) haben eine besonders lange Halbwertszeit. Vergisst der Patient eine Tabletteneinnahme, dann bleiben an diesem Tag 91\% der Blutdrucksenkung erhalten, ein signifikanter Vorteil gegenüber anderen Antihypertensiva-Kombinationen.

\section{- Dr. Dirk Einecke}

Quelle: Pressekonferenz „Rundumschutz mit Aliskiren und Amlodipin - neue Kombinationstablette für süße und schwere Typen", ACC, New Orleans (Veranstalter: Novartis)

\section{Kurz notiert}

Remodeling der Atemwege bei Asthma Der mit $30000 €$ dotierte „International Klosterfrau Award for Research of Airway Diseases in Childhood 2011" wurde an Dr. TillieLouise Hackett, Vancouver (Kanada), verliehen. Der Wissenschaftlerin gelang der Nachweis, dass sich mit TGF- $\beta 1$ in Lungenepithelzellen eine epitheliale-mesenchymale Transition (EMT) induzieren lässt. Für Asthma scheint die Akkumulation von Fibroblasten in der Lunge von großer pathogenetischer Bedeutung zu sein. Sie induzieren u. a. die Synthese und Sekretion extrazellulärer Matrixproteine. Durch die EMT wird die Zahl der Fibroblasten im Lungengewebe erhöht und damit möglicherweise das Remodeling vorangetrieben.

Klosterfrau

\section{Neue hydroaktive Wundauflage} - Das neue Wundpflaster Urgotül ${ }^{\circledR}$ Lite Border lässt sich besonders gut fixieren und passt sich gut den anatomischen Gegebenheiten an. Aufgrund seiner Eigenschaften ist es auch für empfindliche Haut geeignet. Es verklebt nicht mit der Wunde und lässt sich ohne Traumatisierung des neugebildeten Gewebes schmerzfrei wechseln. Auch der Haftrand ist gut hautverträglich. Die Wundauflage basiert auf der bewährten Lipidokolloid-Technologie (TLC) und kann bei schwach nässenden akuten und bei chronischen Wunden angewendet werden.

Urgo $\mathrm{GmbH}$ 